\title{
CHAOS VIA PERIOD-DOUBLING OSCILLATIONS IN AN EXCITON-CARRIER-PHOTON SYSTEM DRIVEN BY OPTICAL PUMPING
}

\author{
NGUYen Ba AN \\ International Center of Condensed Matter Physics, University of Brasilia \\ Caixa Postal 04667, 70919-900 Brasilia-DF, Brazil
}

(Received August 23, 1993)

\begin{abstract}
A detailed stability analysis is performed for a highly excited semiconductor modelled as an exciton-electron-hole-photon system driven externally by optical pumping. Chaotic self-pulsations are shown to occur on both the upper and lower branches of the bistable steady state curve of the system via a sequence of period-doubling oscillation process. Such a route to chaos is well known in laser physics and in other nonlinear systems but not in highly excited semiconductors yet.

PACS numbers: 42.65.-k, 71.35.+z, 42.50.Lc
\end{abstract}

\section{Introduction}

Since the publication of the papers by McCall [1], Bonifacio and Lugiato [2], and Ikeda [3] a new tendency of research has emerged which deals with optical instabilities and spontaneous pulsations in bistable systems. A lot of works in laser physics $[4,5]$ and quantum optics [6] have been devoted to these topics using different model systems and nonlinear processes: two-level (three-level) atom systems [1-3(7)], molecular gas [8], second harmonic (subharmonic) generation $[9,10(11)]$, four-wave mixing $[12,13]$, etc. Recently, semiconductor physicists have also joined the problem [14-18]. Optical turbulence due to exciton-exciton and exciton-biexciton transitions in semiconductors was studied in Refs. [14] to [16]. In Ref. [17] an exciton-carrier system was considered in which the carrier appeared due to exciton ionization. Reference [18] dealt with another mechannism of creating the carrier which may coexist with the exciton. Though in the above-cited papers (Refs. [14] to [18]) irregular self-pulsations were shown to occur, routes to chaos were not clarified yet.

In this paper we reconsider the externally driven exciton-carrier-photon system as in Ref. [18] but the emphasis will be paid to the stability of the steady solutions of the system. The main results of this work [19] have been presented at the XVI Encontro Nacional de Fisica da Materia Condensada held in Caxambu from 18 to 22 May, 1993. 


\section{Dynamic equations}

With the use of optical pumping techniques one can prepare highly excited semiconductors in which electrons, holes, excitons, biexcitons, trions, etc. may coexist under certain circumstances. Construction of phase diagrams for such systems is very complicated and still not solved up to now. In Ref. [20] a qualitative theory of phase transitions was developed for a simpler system consisting only of electrons, holes and excitons. In reality, the exciton is directly generated by a resonant pump pulse, while the electron and the hole may be supplied by doping. If one includes into consideration also the intracrystal photons [21] which inevitably appear inside the semiconductor via the mutual exciton-photon conversion, the IIamiltonian of the resulting exciton-electron-hole-photon system driven by an external pump field of amplitude $\mathcal{E}_{k}$ and frequency $\omega_{k}$ can be written in the form $(\hbar=c=1)$ :

$$
\begin{aligned}
& H=\sum_{\boldsymbol{q}}\left[E_{a}(q) a_{\boldsymbol{q}}^{+} a_{\boldsymbol{q}}+E_{b}(q) b_{\boldsymbol{q}}^{+} b_{\boldsymbol{q}}+E_{e}(q) e_{\boldsymbol{q}}^{+} e_{\boldsymbol{q}}+E_{h}(q) h_{\boldsymbol{q}}^{+} h_{\boldsymbol{q}}\right] \\
& +\mathrm{i} \sqrt{g V}\left[\mathcal{E}_{k} \mathrm{e}^{-\mathrm{i} \omega_{k} t} a_{k}^{+}+\text {h.c. }\right]+G_{1} \sum_{\boldsymbol{q}}\left(a_{\boldsymbol{q}}^{+} b_{q}+b_{\boldsymbol{q}}^{+} a_{\boldsymbol{q}}\right) \\
& +\frac{1}{V} \sum_{p q l}\left[G_{2} a_{p}^{+} a_{q}^{+} a_{q-l^{b}} p_{+l}+G_{3} a_{p}^{+} e_{q}^{+} e_{q-l^{b} p_{+l}}+G_{4} a_{p}^{+} h_{q}^{+} h_{q-l^{b}} p_{+l}+\text { h.c. }\right]
\end{aligned}
$$

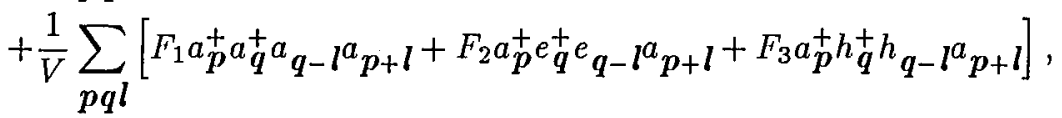

where $E_{a}, E_{b}, E_{e}$ and $E_{h}$ are energies of excitons, intracrystal photons, electrons and holes whose operators are denoted by $a\left(a^{+}\right), b\left(b^{+}\right), e\left(e^{+}\right)$and $h\left(h^{+}\right)$, respectively. $V$ is the volume, $g$ and $G_{1}$ describe the coupling of the exciton with the external field and the intracrystal photon. $G_{2}\left(G_{3}, G_{4}\right)$ stands for the so-called exciton-assisted (electron-assisted, hole-assisted) photon-exciton transition arising due to nonboson character of the exciton at high density. $F_{1}\left(F_{2}, F_{3}\right)$ represents the exciton-exciton (exciton-electron, exciton-hole) interaction. $F_{2}$ and $F_{3}$ describe the interaction between a dipole and a charge. They are about three times larger than $F_{1}$ which is of dipole-dipole-like nature. Analytical expressions of the above-mentioned coupling constants can be found e.g. in Refs. [20] and [21].

Being interested only in the coherent excitons/photons of the momentum/wave vector $k$, we can get from (1) the following equations of motion for the exciton/photon operator a veraged over the coherent state:

$$
\begin{aligned}
\frac{\mathrm{d}}{\mathrm{d} t}\langle a\rangle & =-\mathrm{i}\left[E_{a}+4 n_{a} F_{1}+n_{e} F_{2}+n_{h} F_{3}-\mathrm{i} \gamma_{a}\right]\langle a\rangle \\
-\mathrm{i} & {\left[G_{1}+2 n_{a} G_{2}+n_{e} G_{3}+n_{h} G_{4}\right]\langle b\rangle+\sqrt{g V} \mathcal{E} \mathrm{e}^{-\mathrm{i} \omega t}, } \\
\frac{\mathrm{d}}{\mathrm{d} t}\langle b\rangle & =-\mathrm{i}\left[E_{b}-\mathrm{i} \gamma_{b}\right]\langle b\rangle-\mathrm{i}\left[G_{1}+2 n_{a} G_{2}+n_{e} G_{3}+n_{h} G_{4}\right]\langle a\rangle .
\end{aligned}
$$

In (2) and (3) we suppress for brevity the writing of the index $k . n_{a}, n_{e}$ and $n_{h}$ are the density of excitons, electrons and holes, respectively. $\gamma_{a}$ and $\gamma_{b}$ denote the 
phenomenological damping of the coherent exciton and photon. We seek solutions of (2) and (3) in the forms

$$
\begin{aligned}
& \langle a(t)\rangle=Q\left[x_{a}(t)+\mathrm{i} y_{a}(t)\right] \mathrm{e}^{-\mathrm{i} \omega t}, \\
& \langle b(t)\rangle=Q\left[x_{b}(t)+\mathrm{i} y_{b}(t)\right] \mathrm{e}^{-\mathrm{i} \omega t}
\end{aligned}
$$

with $Q=\left(V \gamma_{a} / F_{1}\right)^{1 / 2} / 2$ being introduced for convenience. Substituting (4), (5) into (2), (3) yields

$$
\begin{aligned}
& \frac{\mathrm{d} x_{a}}{\mathrm{~d} \tau}=-x_{a}-\left(a_{1}-a_{2}-x_{a}^{2}-y_{a}^{2}\right) y_{a}+\left[a_{3}+a_{4}+a_{5}\left(x_{a}^{2}+y_{a}^{2}\right)\right] y_{b}+P, \\
& \frac{\mathrm{d} y_{b}}{\mathrm{~d} \tau}=-y_{a}+\left(a_{1}-a_{2}-x_{a}^{2}-y_{a}^{2}\right) x_{a}-\left[a_{3}+a_{4}+a_{5}\left(x_{a}^{2}+y_{a}^{2}\right)\right] x_{b}, \\
& \frac{\mathrm{d} x_{b}}{\mathrm{~d} \tau}=-b_{1} x_{b}-b_{2} y_{b}+\left[a_{3}+a_{4}+a_{5}\left(x_{a}^{2}+y_{a}^{2}\right)\right] y_{a}, \\
& \frac{\mathrm{d} y_{b}}{\mathrm{~d} \tau}=-b_{1} y_{b}+b_{2} x_{b}-\left[a_{3}+a_{4}+a_{5}\left(x_{a}^{2}+y_{a}^{2}\right)\right] x_{a} .
\end{aligned}
$$

The dimensionless quantities entering (6) to (9) are defined as $\tau=\gamma_{a} t, a_{1}=$ $\left(\omega-E_{a}\right) / \gamma_{a}, a_{2}=2 n F / \gamma_{a}, a_{3}=G_{1} / \gamma_{a}, a_{4}=2 n G / \gamma_{a}, a_{5}=G_{2} / 2 F_{1}, b_{1}=\gamma_{b} / \gamma_{a}$, $b_{2}=\left(\omega-E_{b}\right) / \gamma_{a}$ and $P=2 \mathcal{E}\left(g F_{1}\right)^{1 / 2} \gamma_{a}^{-3 / 2}$, where for simplicity we assume $n_{e}=$ $n_{h}=n, F_{2}=F_{3}=F$ and $G_{3}=G_{4}=G$. Equations (6) to (9) are the dynamic equations of the system under investigation whose steady solutions' stability will be analyzed in the next section for a constant input $P$. For several types of time-dependent inputs, $P=P(t)$, causing switching processes, an analytically rigorous treatment has been developed in Ref. [22].

\section{Stability analysis}

In the steady regime Eqs. (6) to (9) reduce to

$$
\begin{aligned}
& x_{a}^{\mathbf{s}}+\alpha y_{a}^{\mathbf{s}}-\beta y_{b}^{\mathbf{s}}=P, \\
& \alpha x_{a}^{\mathbf{s}}-y_{a}^{\mathbf{s}}-\beta x_{b}^{\mathrm{s}}=0, \\
& \beta y_{a}^{\mathbf{s}}-b_{1} x_{b}^{\mathbf{s}}-b_{2} y_{b}^{\mathbf{s}}=0, \\
& \beta x_{a}^{\mathbf{s}}-b_{2} x_{b}^{\mathbf{s}}+b_{1} y_{b}^{\mathbf{s}}=0,
\end{aligned}
$$

where $\alpha=a_{1}-a_{2}-N_{a}, \beta=a_{3}+a_{4}+a_{5} N_{a}$ and $N_{a}=\left(x_{a}^{\mathrm{s}}\right)^{2}+\left(y_{a}^{\mathrm{s}}\right)^{2}$. The superscript $\mathrm{s}$ specifies the solutions in the steady regime. Equations (10) to (13) are not a set of linear equations because $\alpha$ and $\beta$ themselves depend on $x_{a}^{\mathrm{s}}$ and $y_{a}^{\mathrm{s}}$ through $N_{a}$. However, we can write their formal solutions as

$$
\begin{aligned}
& x_{a}^{\mathbf{s}}=\frac{P \beta\left(\alpha b_{1}+b_{2}\right)}{\Delta}, \\
& y_{a}^{\mathbf{s}}=-\frac{P \beta\left(\beta^{2}+b_{1}-\alpha b_{2}\right)}{\Delta}, \\
& x_{b}^{\mathbf{s}}=\frac{P\left(\beta^{2}+\alpha^{2} b_{1}+b_{1}\right)}{\Delta},
\end{aligned}
$$




$$
y_{b}^{\mathrm{s}}=-\frac{P\left(\alpha \beta^{2}-\alpha^{2} b_{2}-b_{2}\right)}{\Delta},
$$

with

$$
\Delta=\left(b_{1}+\beta^{2}\right)^{2}+\alpha^{2}\left(b_{1}^{2}+b_{2}^{2}\right)+b_{2}^{2}-2 \alpha \beta^{2} b_{2}:
$$

Since the right hand sides of (14)-(17) depend on the yet unknown solutions only through the combination $\left(x_{a}^{\mathbf{s}}\right)^{2}+\left(y_{a}^{\mathrm{s}}\right)^{2}=N_{a}$, they are helpful to determine $x_{a}^{\mathrm{s}}$, $y_{a}^{\mathrm{s}}, x_{b}^{\mathrm{s}}$ and $y_{b}^{\mathrm{s}}$ from known $P$ and $N_{a}$. This will be used later to numerically solve the differential equations (6)-(9). From (14) to (17) we can derive the relations between $I=|P|^{2}, N_{a}$ and $N_{b}=\left(x_{b}^{\mathrm{s}}\right)^{2}+\left(y_{b}^{\mathrm{s}}\right)^{2}$ as follows:

$$
\begin{aligned}
& I=\frac{N_{a}\left(A N_{a}^{4}+B N_{a}^{3}+D N_{a}^{2}+K N_{a}+L\right)}{\left(a_{3}+a_{4}+a_{5} N_{a}\right)^{2}}, \\
& N_{b}=\frac{N_{a}\left[\left(a_{1}-a_{2}-N_{a}\right)^{2}+1\right]}{\left(a_{3}+a_{4}+a_{5} N_{a}\right)^{2}},
\end{aligned}
$$

where

$$
\begin{aligned}
A= & a_{5}^{4}, \quad B=2 a_{5}^{2}\left[b_{2}+2 a_{5}\left(a_{4}-a_{3}\right)\right], \\
D= & b_{1}^{2}+2 a_{5}^{2}\left[b_{1}+\left(a_{4}-a_{3}\right)^{2}+b_{2}\left(a_{2}-a_{1}\right)\right]+\left[b_{2}+2 a_{5}\left(a_{4}-a_{3}\right)\right]^{2}, \\
K= & 2\left\{\left[2 a_{5}\left(a_{4}-a_{3}\right)-b_{2}\right]\left[b_{2}\left(a_{1}-a_{2}\right)+b_{1}+\left(a_{4}-a_{3}\right)^{2}\right]\right. \\
& \left.+b_{1}\left[b_{2}+b_{1}\left(a_{2}-a_{1}\right)\right]\right\}, \\
L= & {\left[b_{1}+\left(a_{4}-a_{3}\right)^{2}+b_{2}\left(a_{2}-a_{1}\right)\right]^{2}+\left[b_{2}-b_{1}\left(a_{2}-a_{1}\right)\right]^{2} . }
\end{aligned}
$$

Equations (19) and (20) say that $N_{b}(\propto$ the photon number) depends on $I$ ( $\propto$ the pump intensity) parametrically through $N_{a}(\propto$ the exciton number). Due to the exciton-assisted mechanism of the exciton-photon transition $\left(\propto G_{2}\right.$, i.e. $\propto a_{5}$ and also $\propto A$ ), Eq. (19) is of the fifth order with respect to $N_{a}$. This might bring about tristability in the system [18]. The $\left(I, N_{a}\right)$-curve can be plotted directly from (19), while for the $\left(I, N_{b}\right)$-curve one needs to use both of (19) and (20). Depending on concrete parameters the shapes of the $\left(I, N_{b}\right)$-curve may be topologically quite different as compared to those of the $\left(I, N_{a}\right)$-curve $[23,18]$. Because of the lack of space, in what follows, we confine ourselves only to a stability analysis of the $\left(I, N_{a}\right)$-curve. The analysis for the $\left(I, N_{b}\right)$-curve gives qualitatively similar results.

To analyze the stability of the steady solutions, let $x_{a, b}^{\mathbf{s}}$ and $y_{a, b}^{\mathbf{s}}$ suffer small perturbations such as

$$
\begin{aligned}
& x_{a, b}^{\mathbf{s}}(\tau)=x_{a, b}^{\mathbf{s}}(0)+\xi_{a, b} \mathrm{e}^{\lambda \tau}, \\
& y_{a, b}^{\mathbf{s}}(\tau)=y_{a, b}^{\mathbf{s}}(0)+\zeta_{a, b} \mathrm{e}^{\lambda \tau},
\end{aligned}
$$

with $x_{a, b}^{\mathbf{s}}(0), y_{a, b}^{\mathbf{s}}(0)$ - the "initial coordinates" of a point lying on the steady state curve $N_{a}=N_{a}(I) ; \xi_{a, b}, \zeta_{a, b}$ - small perturbations and $\lambda$ - a constant characterizing the stability of the point interested. Putting (22), (23) into (6) to (9), we obtain four equations for $\xi_{a, b}$ and $\zeta_{a, b}$ :

$$
\left(\lambda+e_{11}\right) \xi_{a}+e_{12} \zeta_{a}+e_{14} \zeta_{b}=0,
$$




$$
\begin{aligned}
& e_{21} \xi_{a}+\left(\lambda+e_{22}\right) \zeta_{a}+e_{23} \xi_{b}=0 \\
& e_{31} \xi_{a}+e_{32} \zeta_{a}+\left(\lambda+e_{33}\right) \xi_{b}+e_{34} \zeta_{b}=0 \\
& e_{41} \xi_{a}+e_{42} \zeta_{a}+e_{43} \zeta_{a}+\left(\lambda+e_{44}\right) \zeta_{b}=0
\end{aligned}
$$

where

$$
\begin{aligned}
& e_{11}=1-2 x_{a}^{\mathrm{s}}\left(y_{a}^{\mathrm{s}}+a_{5} y_{b}^{\mathrm{s}}\right), \quad e_{12}=\alpha-2 y_{a}^{\mathrm{s}}\left(y_{a}^{\mathrm{s}}+a_{5} y_{b}^{\mathrm{s}}\right), \quad e_{14}=\beta, \\
& e_{21}=-\alpha+2 x_{a}^{\mathrm{s}}\left(x_{a}^{\mathrm{s}}+a_{5} x_{b}^{\mathrm{s}}\right), \quad e_{22}=1+2 y_{a}^{\mathrm{s}}\left(x_{a}^{\mathrm{s}}+a_{5} x_{b}^{\mathrm{s}}\right), \quad e_{23}=-\beta, \\
& e_{31}=-2 a_{5} x_{a}^{\mathbf{s}} y_{a}^{\mathrm{s}}, \quad e_{32}=\beta-2 a_{5} y_{a}^{\mathrm{s}}, \quad e_{33}=b_{1}, \quad e_{34}=b_{2}, \\
& e_{41}=2 a_{5}\left(x_{a}^{\mathrm{s}}\right)^{2}-\beta, \quad e_{42}=2 a_{5} x_{a}^{\mathrm{s}} y_{a}^{\mathrm{s}}, \quad e_{43}=-b_{2}, \quad e_{44}=b_{1} . \quad(28)
\end{aligned}
$$

The constraint for the system (24)-(27) to have nontrivial solutions leads to the characteristic equation determining $\lambda$ :

$$
\lambda^{4}+c_{1} \lambda^{3}+c_{2} \lambda^{2}+c_{3} \lambda+c_{4}=0
$$

with $(i, j, k, l=1 \div 4)$ :

$$
\begin{aligned}
c_{1}= & \sum_{i} e_{i i}, \quad c_{2}=\frac{1}{2} \sum_{i \neq j}\left(e_{i i} e_{j j}-e_{i j} e_{j i}\right), \\
c_{3}= & \sum_{i \neq j \neq k}\left(\frac{1}{6} e_{i i} e_{j j} e_{k k}+\frac{1}{3} e_{i j} e_{j k} e_{k i}-\frac{1}{2} e_{i i} e_{j k} e_{k j}\right), \\
c_{4}= & \frac{1}{2} \sum_{i \neq j \neq k \neq l}\left\{\frac { 1 } { 8 } e _ { i i } e _ { j j } e _ { k k } e _ { l l } \left[e_{i i}\left(e_{j k} e_{l j}-\frac{1}{2} e_{j j} e_{l k}\right)\right.\right. \\
& \left.\left.-\frac{1}{2} e_{i j}\left(e_{j k} e_{l i}-\frac{1}{2} e_{j i} e_{l k}\right) e_{k l}\right]\right\} .
\end{aligned}
$$

The necessary and sufficient conditions for the solutions of the system (10)-(13) to be stable are the negativity of the real parts of all the roots of Eq. [29]. This in turn is obtained from the Hurwitz criteria which require simultaneous fulfilment of the following unequalities:

$$
c_{1}>0, \quad c_{4}>0, \quad c_{1} c_{2}>c_{3} \text { and }\left(c_{1} c_{2}-c_{3}\right) c_{3}>c_{1}^{2} c_{4} .
$$

To test the Iurwitz criteria we first take for simplicity $a_{2}=a_{4}=a_{5}=0$ and following Ref. [15] use $a_{1}=118, a_{3}=23.6, b_{1}=10$ and $b_{2}=0$. For the chosen parameters, the exciton number, $N_{a}$, is plotted as a function of pumping intensity, $I=|P|^{2}$, in Fig. 1 which exhibits optical bistability. Using (19), (20) and (14)-(17) we can check the validity of the Hurwitz criteria (31) for every point of the curve $N_{a}=N_{a}(I)$. Numerical calculations show that the criteria (31) are violated not only on the middle branch (long-dashed in Fig. 1) but also on some domains (short-dashed in Fig. 1) of both the upper and lower branches near the turning points. To explore the nature of the instability in the above-mentioned unstable domains, one needs to follow the time evolution of the system when it is slightly removed from its steady state. With the aid of (19) and (14)-(17), we are able first to determine the "initial coordinates" $x_{a, b}(0), y_{a, b}(0)$ of a point to be 


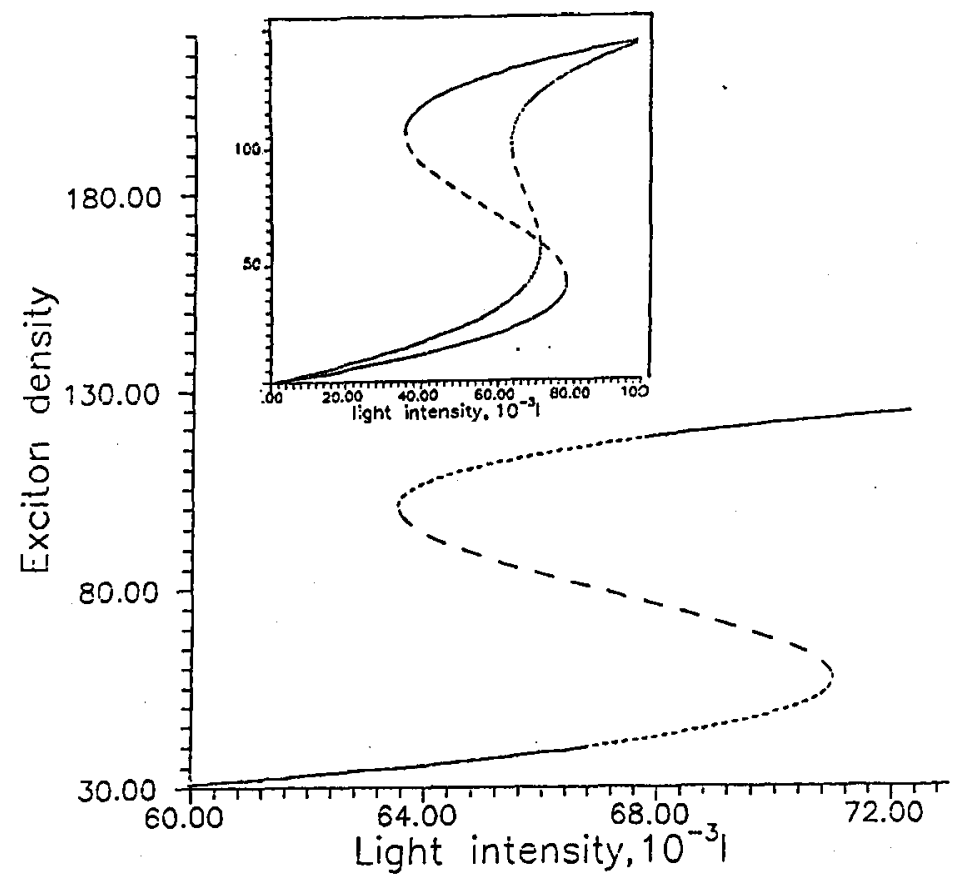

Fig. 1. Exciton density $\left(\propto N_{a}\right)$ versus pump intensity $\left(\propto I=|P|^{2}\right)$ for $a_{1}=118$, $a_{2}=23.6, b_{1}=10, b_{2}=0$ and $a_{2}=a_{4}=a_{5}=0$. The inset: curve with larger (smaller) hysteresis loop is for the case when the carrier is present, $a_{2}=7.5, a_{4}=6$ (absent, $a_{2}=a_{4}=0$ ).

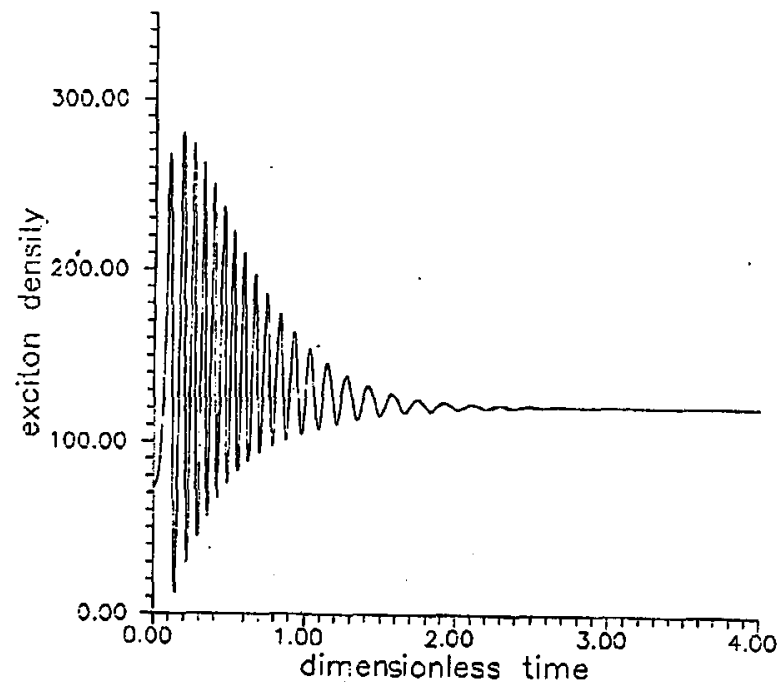

Fig. 2. Time evolution of exciton density for $10^{-3} I=68.6440$. The solution is stable. 


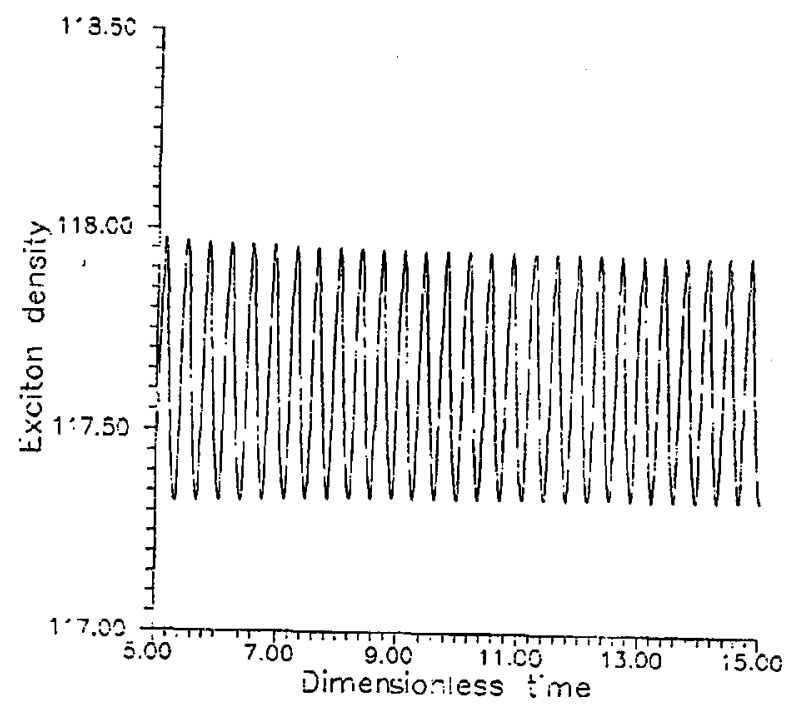

Fig. 3. Periodic oscillation of exciton density for $10^{-3} I=67.1946$.

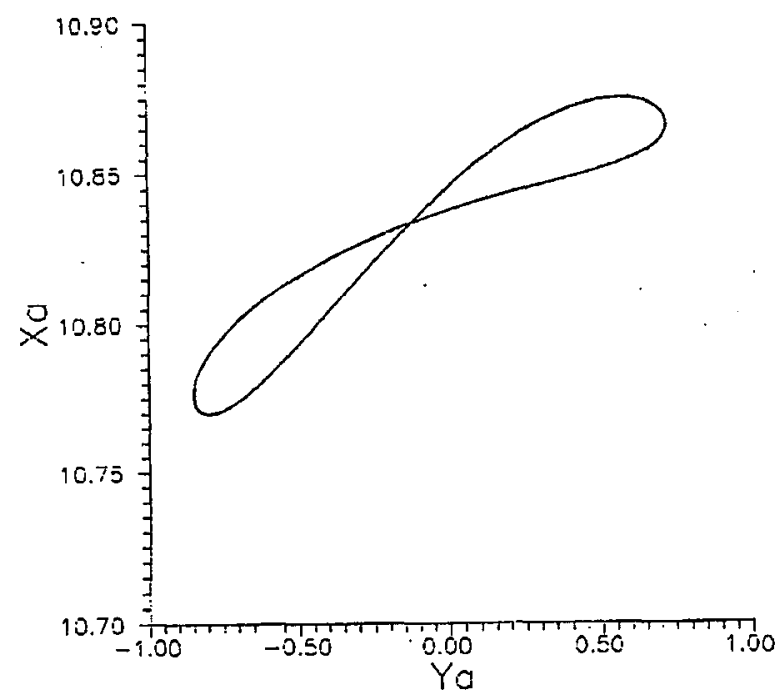

Fig. 4. Twofold limit cycle in the phase space of period-2 oscillations for $10^{-3} I=$ 67.0315 .

checked lying near the steady state curve and then to perform numerical solution of Eqs. (6) to (9). Numerical simulations show that the whole middle branch of the curve $N_{a}=N_{a}(I)$ in Fig. 1 is unstable in the sense that any slight removal from it relaxes to the steady point corresponding to the same input intensity on the upper (lower) branch if the initial removal direction is up (down). For $P=262$ (i.e. $10^{-3} I=68.6440$ ) and the initial state is near the upper branch, the time 


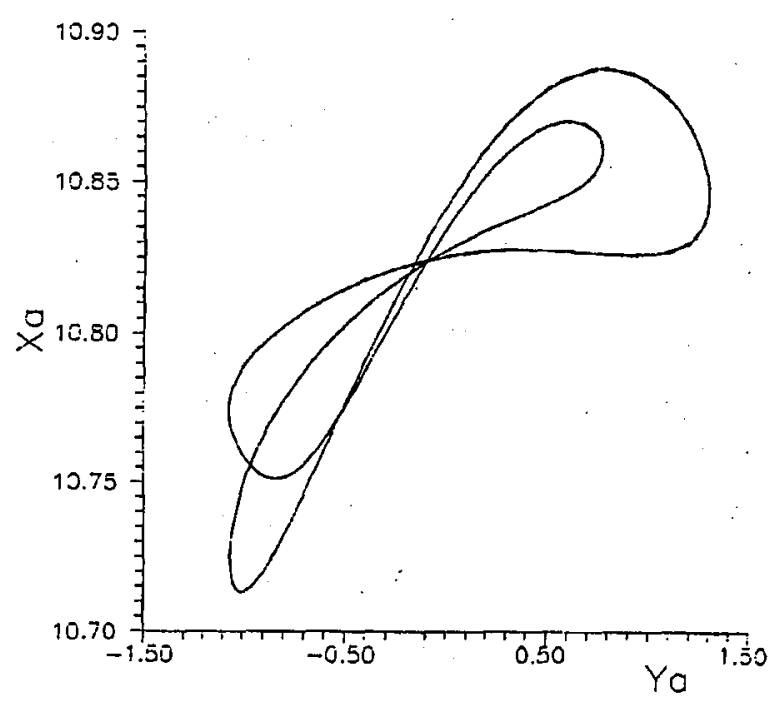

Fig. 5. Fourfold limit cycle in the phase space of period-4 oscillations for $10^{-3} I=$ 66.8609 .

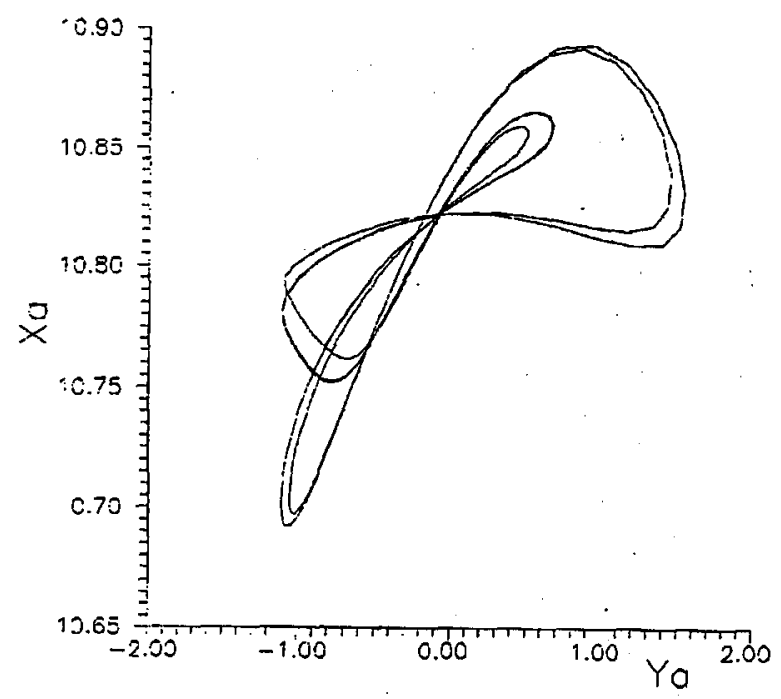

Fig. 6. Eightfold limit cycle in the phase space of period-8 oscillations for $10^{-3} I=$ 66.8130 .

evolution first undergoes some oscillations and then quickly relaxes back to the corresponding steady point in the upper branch (see Fig. 2). Such a point is thus stable. This behavior holds also for larger values of $P$ revealing that the upper branch is stable for $P>262$. For lower values of $P$ a rich variety of instabilities arises. For $P=259.2193$ (i.e. $10^{-3} I=67.1946$ ), the time evolution turns out to 

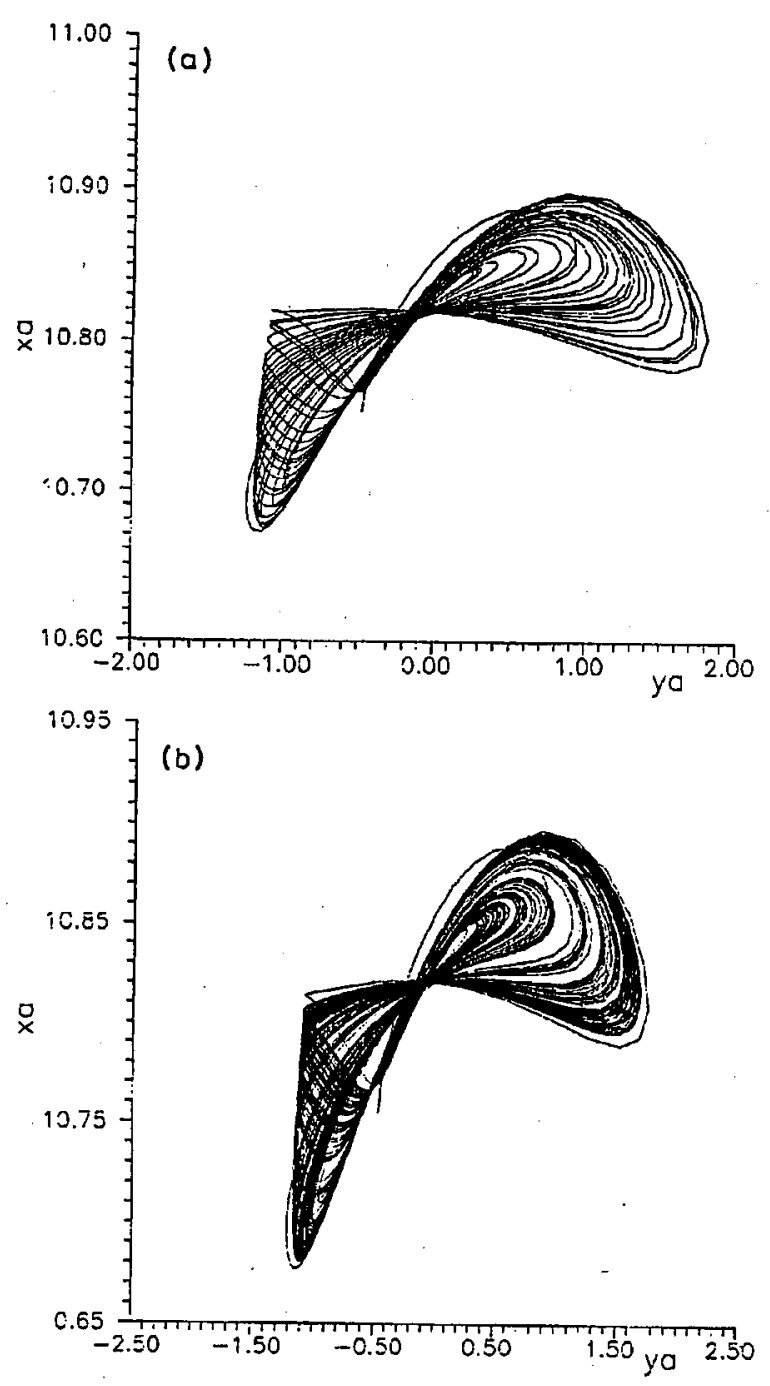

Fig. 7. Chaotic phase portrait for $10^{-3} I=66.8011$ for $\tau=10$ (a) and 60 (b).

be periodic (see Fig. 3). The period of oscillations is suddenly doubled when $P$ is . decreased to 258.9044 (i.e. $10^{-3} I=67.0315$ ). This period-2 oscillation is best seen from a phase portrait drawn in Fig. 4 in the $\left(x_{a}, y_{a}\right)$ plane where the phase trajectory is a twofold limit cycle. When $P$ reaches 258.5747 (i.e. $10^{-3} I=66.8609$ ), the period-2 oscillation disappears and, instead, period-4 oscillations appear which in turn are replaced by period- 8 oscillations at $P=258.4822$ (i.e. $10^{-3} I=66.8130$ ). The phase trajectories of the period- 4 and period- 8 oscillations are represented in Figs. 5 and 6 , respectively. When $P$ is falling on a small interval between 258.2992 

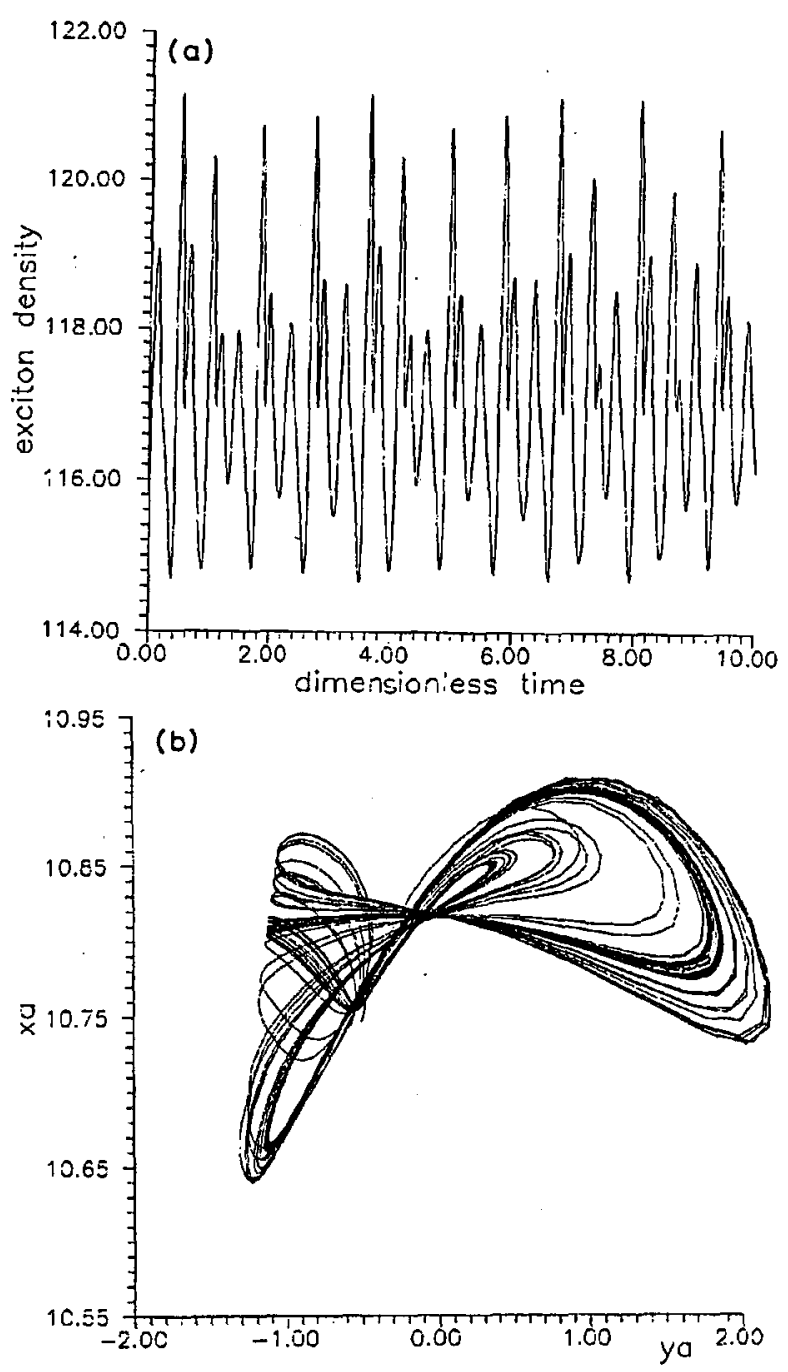

Fig. 8. Chaotic behavior for $10^{-3} I=66.7185$ : (a) time evolution and (b) phase portrait for $\tau=60$.

and 258.4591 (i.e. $10^{-3} I=66.7185 \div 66.8011$ ), we observe chaotic self-pulsations. The phase portrait of chaos at $P=258.4591$ is traced in Figs. $7 \mathrm{a}$ and $7 \mathrm{~b}$ respectively for $\tau=10$ and 60 . We see that the trajectory is not a limit cycle and, for a long time, it is going to fill in completely a finite area in the phase space indicating the occurrence of chaos. In Figs. $8 \mathrm{a}$ and $8 \mathrm{~b}$ we plot the irregular self-pulsations for $P=258.2992$ as a function of time and as in the phase space for $\tau=60$. Between the window of chaos and the turning point the oscillations display themselves in the form of a chain of identical multi-peaked pulses whose phase portraits 


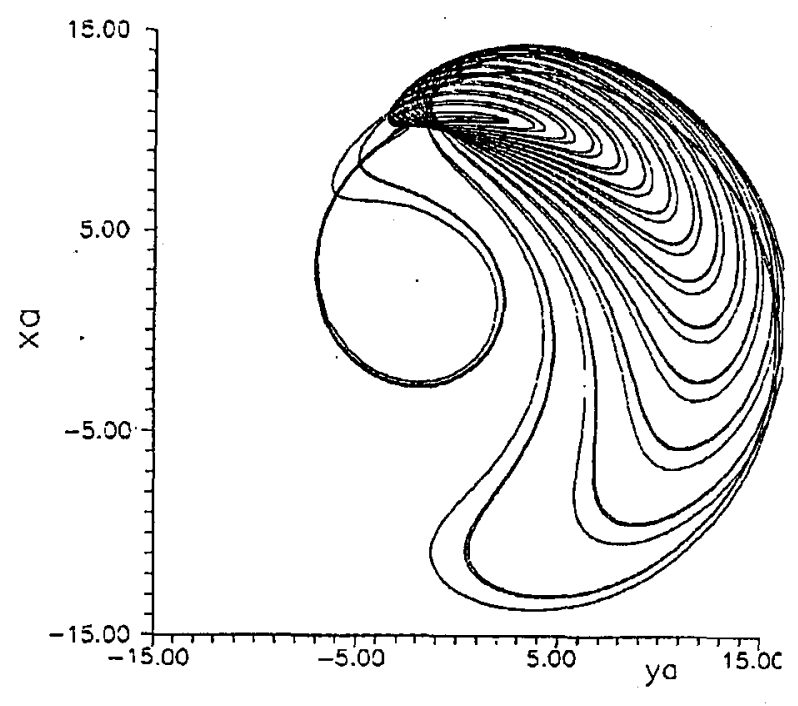

Fig. 9. Multi-fold limit cycle in the phase space for $10^{-3} I=66.3636$.

look quite complicated but are in fact limit cycles. For example, Fig. 9 illustrates a multi-fold limit cycle for $P=258.6630$ (i.e. $10^{-3} I=65.3636$ ) lying in the just said interval. Similar behaviors also hold for the lower branch of the curve $N_{a}=N_{a}(I)$ if the control parameter, $P$, is however increasing to approach the other turning point from below.

To study the carrier-induced modification we represent simultaneously two curves on the inset of Fig. 1. The one with smaller hysteresis loop is the same as that in Fig. 1. The other one, with larger hysteresis loop, is for the case of $a_{2}=7.5$ and $a_{4}=6$, i.e. when a small density of carriers is present together with the exciton. A stability analysis can also be performed for the latter case. The result is that for the whole range of $P$ where the curve with no carriers suffers different kinds of instabilities as described above, the curve with carriers present is stable. This feature might provide a means of controlling chaos by doping in bistable systems especially when these are intended to be used as memory elements.

\section{Conclusion}

In conclusion, we have studied the sensitivity of the transient behavior of an interacting exciton-carrier-photon system driven by an external optical pump to the initial condition and to the control parameters. Chaotic self-pulsations in such systems are demonstrated to occur via a period-doubling cascade when the pump intensity is approaching the turning points. It has been shown that the bistable system can be controlled by optical pumping as well as by $n$ - and/or $p$-type doping (for influence of donors on excitonic optical bistability see e.g. Ref. [24]). 


\section{Acknowledgments}

This work was financially supported by Conselho National de Desenvolvimento Cientifico e Tecnologico (CNPq). The author wishes to thank CNPq for a visiting fellowship, Prof. A. Ferraz for the hospitality at the Centro Internacional de Fisica da Materia Condensada (Universidade de Brasilia) and the organizers of the XVI Encontro Nacional da Fisica Condensada, for making his participation in the meeting possible.

\section{References}

[1] S.L. McCall, Appl. Phys. Lett. 32, 284 (1978).

[2] R. Bonifacio, L.A. Lugiato, Lett. Nuovo Cimento 21, 510 (1978).

[3] K. Ikeda, Opt. Commun. 30, 257 (1979).

[4] F.T. Arecchi, Phys. Scr. 36, 911 (1987).

[5] Dynamical Instabilities and Pulsations in Lasers, Ed. E. Wolf, Progress in Optics XXV, Elsevier, 1988.

[6] Instabilities and Chaos in Quantum Optics II, Ed. N.B. Abraham, F.T. Arecchi, L.A. Lugiato, Plenum Publ., 1988.

[7] C.M. Savage, H.J. Carmichael, D.F. Walls, Opt. Commun. 42, 211 (1982).

[8] W.J. Firth, R.G. Harrinson, I.A. Al-Saidi, Phys. Rev. A 33, 2449 (1986).

[9] P. Mandel, T. Erneux, Optica Acta 29, 7 (1982).

[10] K. Grygiel, P. Szlachetka, Opt. Commun. 78, 177 (1990).

[11] N.P. Pettiaux, Li Ruo-Ding, P. Mandel, in: Coherence and Quantum Optics VI, Ed. J.II. Eberly, Plenum Press, New York 1990.

[12] P. Mandel, N.P. Pettiaux, Wang Kaige, P. Galatola, L.A. Lugiato, Phys. Rev. A 43, 424 (1991).

[13] N.V. Alekseeva, K.N. Alekseev, V.A. Balueva, G.P. Berman, A.K. Popov, V.Z. Yakhin, Opt. Quantum Electron. 23, 603 (1991).

[14] A.H. Rotaru, Fiz. Tverd. Tela 28, 2492 (1986).

[15] S.A. Moskalenko, A.H. Rotaru, V.A. Zaloj, Phys. Status Solidi B 150, 401 (1988).

[16] B.Sh. Parkanskii, A.H. Rotaru, Zh. Eksp. Teor. Fiz. 99, 899 (1991).

[17] M. Mareyen, F.J. Schutte, R. Tiebel, Phys. Status Solidi B 159, 235 (1990).

[18] Nguyen Ba An, Preprint ICTP, Trieste, IC/91/172, 1991.

[19] Nguyen Ba An, in: Fisica da Materia Condensada: Programa e Resumos, S.B.F., Sao Paulo 1993, p. 256.

[20] II. Haug, Z. Phys. B 24, 351 (1976).

[21] S.A. Moskalenko, Vvedenie v teoriu eksitonov bolshoi plotnosti, Shtiinxa, Kishinev 1983.

[22] Nguyen Ba An, P. Mandel, J. Phys., Condens. Matter 5, 4451 (1993).

[23] Nguyen Ba An, Le Thi Cat Tuong, Solid State Commun. 76, 1139 (1990).

[24] Nguyen Ba An, Le Thi Cat Tuong, Can. J. Phys., 70, 1222 (1992). 